\title{
Deep GMRT images of the Spitzer extragalactic First Look Survey at $610 \mathrm{MHz}$
}

\author{
David A. Green and Timothy S. Garn
}

\author{
Cavendish Laboratory, 19 J.J. Thomson Avenue, Cambridge, CB4 3PT, UK \\ email: dag@mrao.cam.ac.uk, tsg25@cam.ac.uk
}

We have observed the Spitzer extragalactic First Look Survey (xFLS) region with the Giant Meterwave Radio Telescope (GMRT), near Pune, India, at $610 \mathrm{MHz}$. Seven pointings were observed with the GMRT, one central and six in a surrounding hexagon. Each field was observed with an integration time of $\sim 200 \mathrm{~min}$., in a series of scans spread over a range of LST to improve $u-v$ coverage. Two sidebands, each of $16 \mathrm{MHz}$ with 128 channels - to allow narrow band interference to be excised efficiently - were observed. The synthesised beam of the images is $\sim 5^{\prime \prime}$, and as the primary beam of the GMRT at $610 \mathrm{MHz}$ is $\sim 43^{\prime}$, these seven pointings cover most of the xFLS region.

Initial comparison of sources detected in the overlap regions between adjacent pointings, and with existing surveys of the region, revealed two problems. $(i)$ The coordinates of the GMRT images were slightly distorted, which was found to be due to slight errors in the timestamps, and hence $(u, v, w)$ coordinates, of the visibilities. (ii) The flux densities of sources seen in adjacent fields varied systematically with position, implying an offset to the effective primary beam of the telescope. After correcting for these problems, the final images have an r.m.s. noise of typically $30 \mu \mathrm{Jy} \mathrm{beam}^{-1}$, before primary beam correction, although dynamic range issues limit the quality of the images near bright sources.

These results will be combined with existing radio data and IR data from the Spitzer Space Telescope, to investigate the far-infrared - radio correlation up to redshifts of about $z=2$. 